\title{
Perturbed period-doubling bifurcation. Il. Experiments on Josephson junctions
}

\author{
Eriksen, Gert Friis; Hansen, Jørn Bindslev
}

Published in:

Physical Review B

Link to article, DOI:

10.1103/PhysRevB.41.4189

Publication date:

1990

Document Version

Publisher's PDF, also known as Version of record

Link back to DTU Orbit

Citation (APA):

Eriksen, G. F., \& Hansen, J. B. (1990). Perturbed period-doubling bifurcation. II. Experiments on Josephson junctions. Physical Review B, 41(7), 4189-4194. https://doi.org/10.1103/PhysRevB.41.4189

\section{General rights}

Copyright and moral rights for the publications made accessible in the public portal are retained by the authors and/or other copyright owners and it is a condition of accessing publications that users recognise and abide by the legal requirements associated with these rights.

- Users may download and print one copy of any publication from the public portal for the purpose of private study or research.

- You may not further distribute the material or use it for any profit-making activity or commercial gain

- You may freely distribute the URL identifying the publication in the public portal

If you believe that this document breaches copyright please contact us providing details, and we will remove access to the work immediately and investigate your claim. 


\title{
Perturbed period-doubling bifurcation. II. Experiments on Josephson junctions
}

\author{
G. Friis Eriksen and J. Bindslev Hansen \\ MIDIT, Physics Laboratory I, The Technical University of Denmark, DK-2800 Lyngby, Denmark
}

(Received 24 May 1989)

\begin{abstract}
We present experimental results on the effect of periodic perturbations on a driven, dynamic system that is close to a period-doubling bifurcation. In the preceding article a scaling law for the change of stability of such a system was derived for the case where the perturbation frequency $\omega_{S}$ is close to the resonances given by $\omega_{S} / \omega_{D}=\frac{1}{2}, \frac{3}{2}, \frac{5}{2}, \ldots$, where $\omega_{D}$ is the driving frequency. The theoretical prediction for the shift of the bifurcation point, $\Delta \mu_{B}$, which we use as a measure of the stabilization, is $\Delta \mu_{B} \sim A_{S}^{2}$, where $A_{S}$ is the perturbation amplitude. We have investigated $\Delta \mu_{B}$ as a function of the frequency and the amplitude of the perturbation signal $\Delta \mu_{B}\left(\omega_{S}, A_{S}\right)$ for a model system, the microwave-driven Josephson tunnel junction, and find reasonable agreement between the experimental results and the theory.
\end{abstract}

\section{INTRODUCTION}

The period-doubling bifurcation is observed in a wide range of biological, chemical, electrical, hydrodynamical, and optical systems. We have studied the stability of a nonlinear electrical device, the microwave-driven Josephson tunnel junction close to a period-doubling bifurcation in the presence of perturbations. ${ }^{1-3}$ The amplifying mechanism in Josephson parametric amplifiers is closely related to this problem.

The theory is introduced in the preceding article, ${ }^{4}$ where it is shown that a near-resonant perturbation signal may stabilize the system against the incipient perioddoubling bifurcation. In this process the perturbation signal itself will be amplified. It is demonstrated how both the amplitude and the frequency of the perturbation affect the stability of the period-doubling bifurcation. It is shown that when the frequency of the periodic perturbation $\omega_{S}$ is close to $m \omega_{D} / 2$ (where $m=1,2,3, \ldots$ and $\omega_{D}$ is the driving frequency) it will change the stability of the system. These resonance frequencies can be divided into two classes: $\omega_{S}=\left(n-\frac{1}{2}\right) \omega_{D}$ and $\omega_{S}=n \omega_{D}$. In the first case the stability is always improved and a scaling law is derived: $\Delta \mu_{B} \sim A_{S}^{2}$, where $\Delta \mu_{B}$ is the shift of the bifurcation point. In the second case, $\omega_{S}=n \omega_{D}$, it is not possible to make any definite prediction, since the perturbation can either stabilize or destabilize the dynamical system (depending on the phase).

We use a Josephson tunnel junction biased by dc and by microwave currents. Experimental data obtained on $\mathrm{Nb}-\mathrm{NbO}_{x}-\mathrm{Pb}$ tunnel junctions of the overlap type are reported and compared with the (analytical) theoretical predictions, which are supported by numerical calculations and analog simulations. ${ }^{4}$ For this comparison we concentrate on the effect of the perturbing signal: the shift of the bifurcation point as a function of the amplitude and frequency of the perturbation, i.e., $\Delta \mu_{B}\left(A_{S}, \omega_{S}\right)$.

We regard this analysis of the nonlinear dynamics as a new way of describing the action of the Josephson parametric amplifier, a microwave device that has attracted renewed interest after recent reports on substantial improvements in performance. 5,6

\section{THEORETICAL MODEL}

Here we shall only present the main theoretical results and refer to Ref. 4 for a full exposition of the theory. The following set of $n$-coupled differential equations are used to model an $n$-dimensional dynamical system:

$\dot{x}=f_{\mu}(x, t)+s(t)+n(t), \quad(x, t) \in \mathcal{R}^{n} \times \mathcal{R}, \mu \in 1$,

where $f_{\mu}(\cdot, t)=f_{\mu}\left(\cdot, t+T_{D}\right)$ is periodic with the period $T_{D}, \mu$ is a control parameter defined in some interval $I$. $s(t)$ and $n(t)$ are a small periodic perturbation signal and a small "white" noise term, respectively. The period of the signal is denoted $T_{S}$ and the corresponding frequency $\omega_{S}=2 \pi / T_{S}$. The unperturbed dynamical system is assumed to be close to a period-doubling bifurcation. By increasing the control parameter, $\mu$, the response will change from a periodic solution with period $T_{D}$ to a periodic solution of period $2 T_{D}$. The value of the control parameter for which this happens is called the bifurcation point, $\mu_{0}$. The theory is valid for $\mu \lesssim \mu_{0}$. The detuning, $\delta$, is defined as

$$
\delta=2\left(\omega_{S} / \omega_{D}\right)-k,
$$

where $k$ is an integer chosen such that

$$
-\frac{1}{2} \leq \delta \leq \frac{1}{2} \text {. }
$$

With this definition the detuning is always measured relative to the nearest resonance frequency, $\omega_{S} / \omega_{D}$ $=\frac{1}{2}, 1, \frac{3}{2}, \ldots$.

Svensmark and Samuelson ${ }^{4}$ have carried out a linear stability analysis and found that for odd values of $k$ (i.e., for $\omega_{S}$ close to $\left.n \omega_{D}, n=0,1,2,3, \ldots\right)$, the following expression is obtained for $\Delta \mu_{B}$, the shift of the original (unperturbed) bifurcation point $(\lambda=-1-\mu)$ :

$$
\Delta \mu_{B}=\mu-\mu_{0} \simeq A_{1} \cos \pi \delta \simeq A_{1}\left[1-(\pi \delta)^{2} / 2\right],
$$

where $A_{1}$ is proportional to the amplitude of the periodic 
perturbation. Since $\Delta \mu_{B}$ depends on the phase of the perturbation (the sign of $A_{1}$ ), no definite conclusion on the change of stability can be made.

On the other hand, for even values $k$, i.e., for $\omega_{S}$ close to $\left(n+\frac{1}{2}\right) \omega_{D}(n=1,2,3, \ldots)$ the perturbation signal at $\omega_{S}$ will always tend to stabilize the system against the incipient period-doubling bifurcation. This may be seen from the expression for the shift of the bifurcation point:

$$
\Delta \mu_{B} \simeq \frac{1}{2} A_{1}^{2} \cos 2 \pi \delta \simeq A_{1}^{2}\left[1-(2 \pi \delta)^{2} / 2\right]
$$

from which it is seen that the stabilization $\left(\Delta \mu_{B}\right)$ is proportional to the square of the amplitude of the perturbation. For increasing detuning $\delta$ the stabilizing effect diminishes, i.e., $\Delta \mu$ decreases for increasing $\delta$.

The other important property of a period-doubling bifurcation is its ability to amplify perturbations [see Eq. (11) of Ref. 4]. The resonance condition is

$$
\lambda^{2}=\left(1+A_{1}^{2}\right) \cos 2 \pi \delta,
$$

yielding

$$
2 \Delta \mu_{R} \simeq A_{1}^{2}-2(\pi \delta)^{2} \text {. }
$$

We note that the resonance in general differs a little from the bifurcation. The period-doubled response will be at $\omega_{D} / 2$, while the resonance will be at $\omega_{S}$. They coincide for small values of $\delta$.

\section{JOSEPHSON JUNCTION MODEL}

A small (zero-dimensional) Josephson tunnel junction may be modeled by the capacitively resistively shunted junction (CRSJ model). In the case of a junction driven by a dc source, two microwave current generators and a noise current source (from the Nyquist current noise in the dissipative quasiparticle tunneling conductance $1 / R$ ) the governing second-order nonlinear differential equation for the junction dynamics is

$\phi_{t t}+\alpha \phi_{t}+\sin \phi=i+a_{D} \sin \omega_{D} t+a_{S} \sin \omega_{S} t+\eta(t)$.

Here the subscripts denote partial differentiation, $\phi$ is the quantum-mechanical phase difference across the junction and $\alpha$ is the damping parameter $\left(\alpha=1 / \sqrt{\beta_{c}}\right.$, where $\beta_{c}$ is the McCumber parameter for the junction, $\left.\beta_{c}=2 e R^{2} C I_{c} / \hbar\right) . I_{c}$ is the critical current of the junction. $a_{D}=A_{D} / I_{C}$ and $a_{S}=A_{S} / I_{c}$ are the normalized amplitudes of the driving and the perturbation signals, respectively. $\omega_{D}$ and $\omega_{S}$ are the corresponding frequencies (normalized to the junction maximum plasma frequency $\omega_{P}=\left(2 e I_{c} / \hbar C\right)^{1 / 2}$ where $C$ is the junction capacitance). $i=I_{\mathrm{dc}} / I_{c}$ is the normalized dc bias and $\eta(t)$ the normalized white noise current. We note that this equation also describes a driven, damped pendulum subjected to two periodic perturbating forces and to a stochastic (Brownian) force term.

In Ref. 4 Svensmark and Samuelsen have presented the results of their numerical calculations of $\Delta \mu_{B}\left(A_{S}, \delta\right)$ based on Eq. (6). The quadratic scaling law predicted by Eq. (3) was indeed found for $\omega_{S} / \omega_{D} \approx \frac{1}{2}$ for three different values of $\delta$ to hold as long as the perturbation amplitude was small (see Fig. 4 in Ref. 4). Above a certain level of perturbation, which depends on the relative detuning $\delta$, the relationship between $\Delta A_{D}$ and $A_{S}$ became almost linear. These numerical results were corroborated by analog simulations.

It was also found that for fixed $\Delta \mu_{B}, A_{S}$ was proportional to $\delta$, i.e., when the perturbation frequency was tuned closer to the frequency of the period-doubled signal, the perturbation amplitude necessary to obtain the same stabilizing effect on the Josephson junction decreased proportionately.

\section{EXPERIMENTAL RESULTS ON JOSEPHSON JUNCTIONS}

\section{A. Experimental technique}

Experimentally, the Josephson tunnel junction between two superconductors is a well-characterized highfrequency nonlinear electrical component that has proven to be exemplary for the study of nonlinear phenomena in general. For the purpose of this study where no spatial dependence is involved, a small (pointlike) Josephson tunnel junction is needed; i.e., the junction dimensions should not exceed the Josephson penetration depth $\lambda_{J}$, which is the characteristic length for spatial variations of the tunneling current. In addition, appropriate broadband coupling between the junction and two oscillators (microwave sources for the driven and perturbation signals) and one detector (of the period-doubled signal) should be provided.

The experimental investigation was carried out on $\mathrm{Nb}$ $\mathrm{NbO}_{\gamma}-\mathrm{Pb}$ tunnel junctions of the overlap type. The junction areas were about $30 \times 30 \mu \mathrm{m}^{2}$ with capacitances around $90 \mathrm{pF}$, maximum plasma frequencies $\omega_{P} / 2 \pi=\left(2 e I_{c} / \hbar C\right)^{1 / 2} / 2 \pi$ in the range $10-25 \mathrm{GHz}$, and Josephson penetration depth $\lambda_{J}=50-120 \mu \mathrm{m}$.

In order to minimize the effect of room-temperature noise and interference from external noise sources on the junction, all leads connected to the junction were carefully shielded and filtered. The ambient magnetic field was reduced to a few milligauss by two concentric shields of high-permeability metal ( $\mu$ metal) around the cryostat. A small magnetic field generated by a copper wire-wound solenoid could be applied in the plane of the junction in order to be able to vary $I_{c}$ and thereby $\omega_{P}$. The entire experimental setup was placed in a rf-shielded room.

The microwave coupling to the junction was established by an inverted microstrip structure (Fig. 1). The glass substrate with the junction and its coupling circuit was mounted in a temperature-stabilized metal box with a large heat capacity, which in turn was placed in a vacuum can immersed in a pressure-regulated liquid-helium bath. The temperature of the junction could be held constant to within $\pm 10 \mu \mathrm{K}$.

The junction was driven by a monochromatic microwave signal, $A_{D} \sin \omega_{D} t$, from a synthesizer source in the frequency range $16-20 \mathrm{GHz}$ (linewidth $<100 \mathrm{~Hz}$ ) coupled to the junction through a direct inverted microstrip line as seen in Fig. 1. A possible period-doubled (half harmonic) signal generated in the junction (near the plas- 

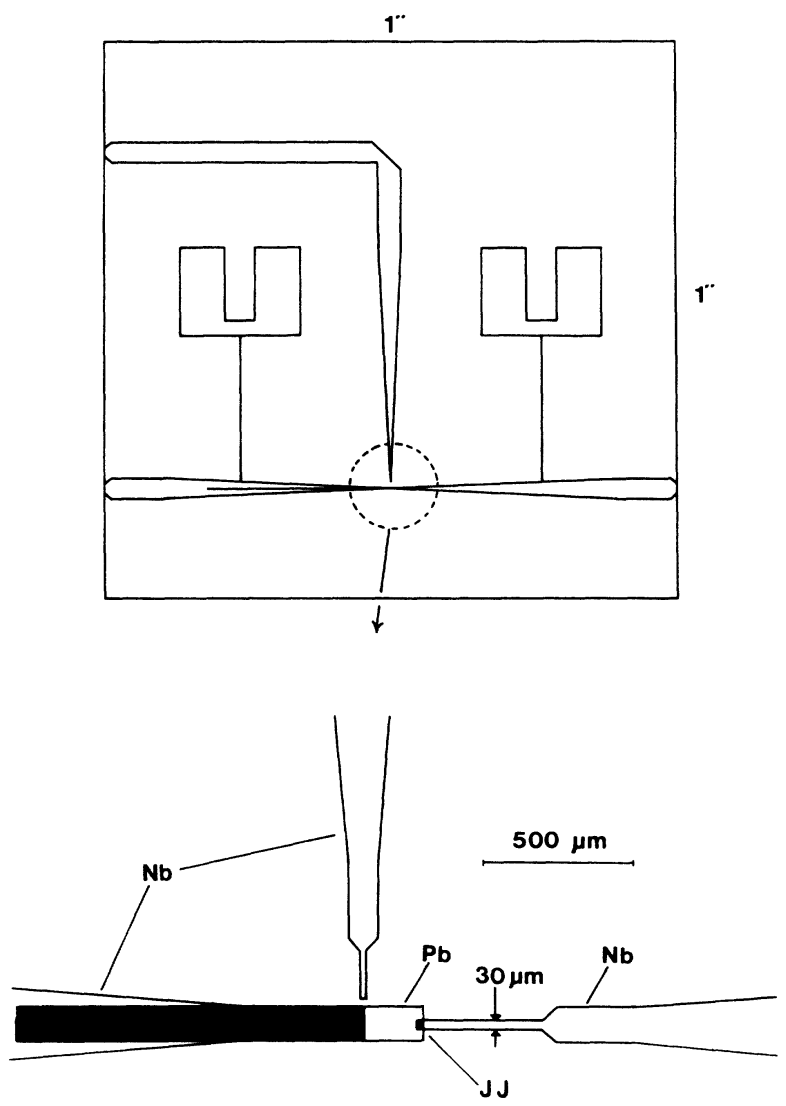

FIG. 1. Sample geometry including the microwave coupling circuit and the overlap $\mathrm{Nb}-\mathrm{NbO}_{x}-\mathrm{Pb} 30 \times 30 \mu \mathrm{m}^{2}$ Josephson tunnel junction. A copper ground plane is placed approximately $400 \mu \mathrm{m}$ above the glass substrate with the $\mathrm{Nb}$ thin-film microstrip circuit. The contact pads for the dc bias leads are also shown.

ma frequency) was coupled out through the same microstrip connection to low-noise field-effect transistor (FET) amplifiers, which was followed by a digital storage spectrum analyzer (with a minimum resolution bandwidth of $100 \mathrm{~Hz}$ ). To avoid saturation of the amplifiers by the pump signal, low-pass filters were inserted between the junction and the amplifier.

The perturbation signal, $A_{S} \sin \omega_{S} t$, generated in a second microwave source, was coupled into the junction via an " $L$ "-shaped microstrip antenna line (see Fig. 1). The frequency of the perturbation signal was slightly detuned (by 50-500 $\mathrm{MHz}$ ) from the period-doubled signal frequency at $\omega_{D} / 2$ (the half harmonic). Since $\omega_{D} / 2 \approx(2 \pi) 10 \mathrm{GHz}$, the relative detuning $\delta$ was therefore in the range $\left(5 \times 10^{-6}\right)-\left(5 \times 10^{-2}\right)$. The linewidth of the perturbation signal was $2 \mathrm{kHz}$.

The microwave coupling to the junction was investigated by using the junction itself as a detector. The suppression of the critical current of the junction was measured as a function of the frequency and the power applied from the two sources. From such measurements we deduced that the microwave coupling both in the vicinity of the pump frequency and of its half harmonic was strongly frequency dependent but always weak. Using a compar- ison between the measured values of the critical current of the junction as a function of the applied microwave power, $I_{c}\left(A_{D}\right)$ and $I_{c}\left(A_{S}\right)$, and the results of numerical simulations based on the resistively shunted junction model including noise [Eq. (6)], we were able to determine the amplitude of the microwave currents through the junction, $A_{D}$ and $A_{S}$, see Fig. 2.

In order to characterize the white noise term $\eta(t)$ in Eq. (6), the effective noise temperature affecting the junction was measured in situ by recording the noiseperturbed distribution of the critical current for a large number of switching events, $P\left(I_{c}\right)$, in a low-noise data acquisition system (the switching was induced by sweeping the dc bias, thereby tracing out the hysteretic $I-V$ curve of the junction). Assuming a white noise spectrum, such a measurement of the noise currents (primarily around the junction plasma frequency) yielded an effective noise temperature of about $10 \mathrm{~K}$ at a bath temperature of $4.2 \mathrm{~K}$.

\section{B. Measurement procedures}

The measurement of the dynamic stabilization of the junction against a period-doubling bifurcation was carried out in the following way.

First, the dc bias, $I_{\mathrm{dc}}$, the magnetic field strength, $B$, and the temperature, $T$, were chosen such that the resonance frequency of the junction lay near $9 \mathrm{GHz}$, where our microwave detection system was most sensitive.

Second, with no applied perturbation signal ( $\left.A_{S}=0\right)$ the amplitude of the drive (pump) signal, $A_{D}$, around 18

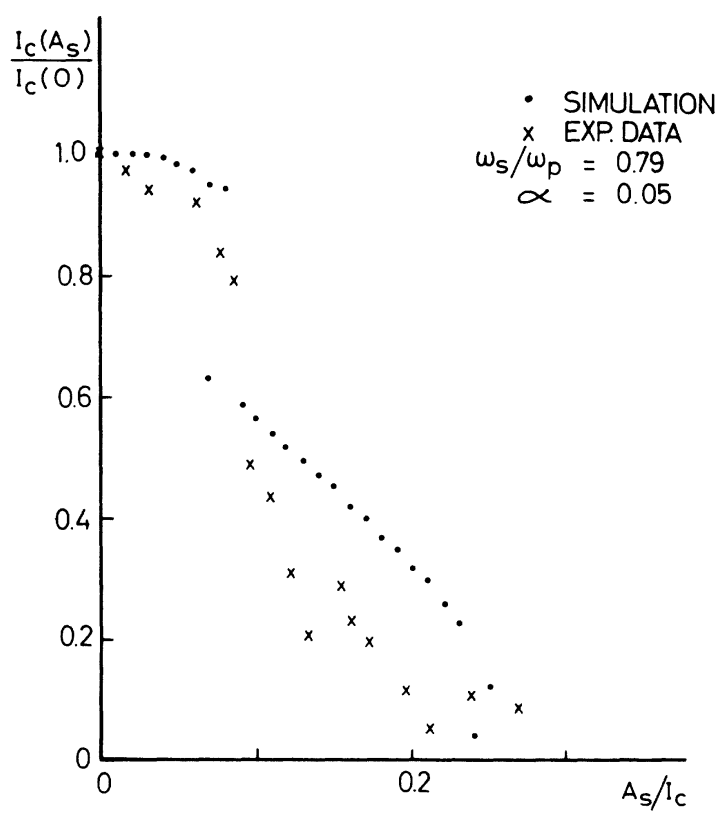

FIG. 2. Experimental and simulated suppression of the critical current vs the normalized microwave current in a junction, in this case for $a_{S}=A_{S} / I_{c}$. The experimental points have been linearly scaled along the horizontal axis to fit to the simulated points around $a_{s}=0.1$. The experimental parameters were $I_{c}=166 \mu \mathrm{A}, \omega_{P} / 2 \pi=12.68 \mathrm{GHz}, \omega_{S} / 2 \pi=9.965 \mathrm{GHz}, \alpha=0.05$ $\left(\beta_{c}=400\right), \quad T_{\text {bath }}=4.2 \mathrm{~K}$, and $T_{\text {eff }}=10 \mathrm{~K} \quad\left(\Gamma=2 e k_{B} T_{\text {eff }} /\right.$ $h I_{c}=0.0030$ ). 
$\mathrm{GHz}$ was varied until a period-doubled signal (half harmonic) was observed at the resonance frequency of the junction. Recordings of the amplitude of the perioddoubled signal, $A\left(\omega_{D} / 2\right)$, as a function of dc bias or drive amplitude were taken.

Third, a near-resonant perturbation signal was applied ( $A_{S}>0$ ) and the suppression of the period-doubled signal was investigated as a function of the detuning $\delta$ and the amplitude $A_{S}$. This was done by recording traces of $A\left(\omega_{D} / 2\right)$ versus $I_{\mathrm{dc}}, A_{D}$, or frequency, while keeping the other parameters constant.

\section{Experimental results}

The stabilizing effect of a near-resonant periodic perturbation was clearly observed. The qualitative features of such an observation are seen in Fig. 3, where amplitude-frequency recordings from the spectrum analyzer are shown as a function of dc bias (for a fixed drive amplitude) both with and without an applied perturbation signal. The main characteristics of such experimental observations are as follows: (1) The perturbation signal suppresses the period-doubled signal (the halfharmonic amplitude), and (2) the perturbation signal is amplified as seen by the appearance of an idler signal at the image frequency $\omega_{i}=\omega_{D}-\omega_{S}\left(=\omega_{D} / 2+\delta\right)$.

We note that the microwave setup used in this work did not provide isolation between the input and the output of the junction, and we have therefore not been able to measure the amplification in the system. The appearance of the idler signal is, however, indicating that the junction is working as an amplifier.
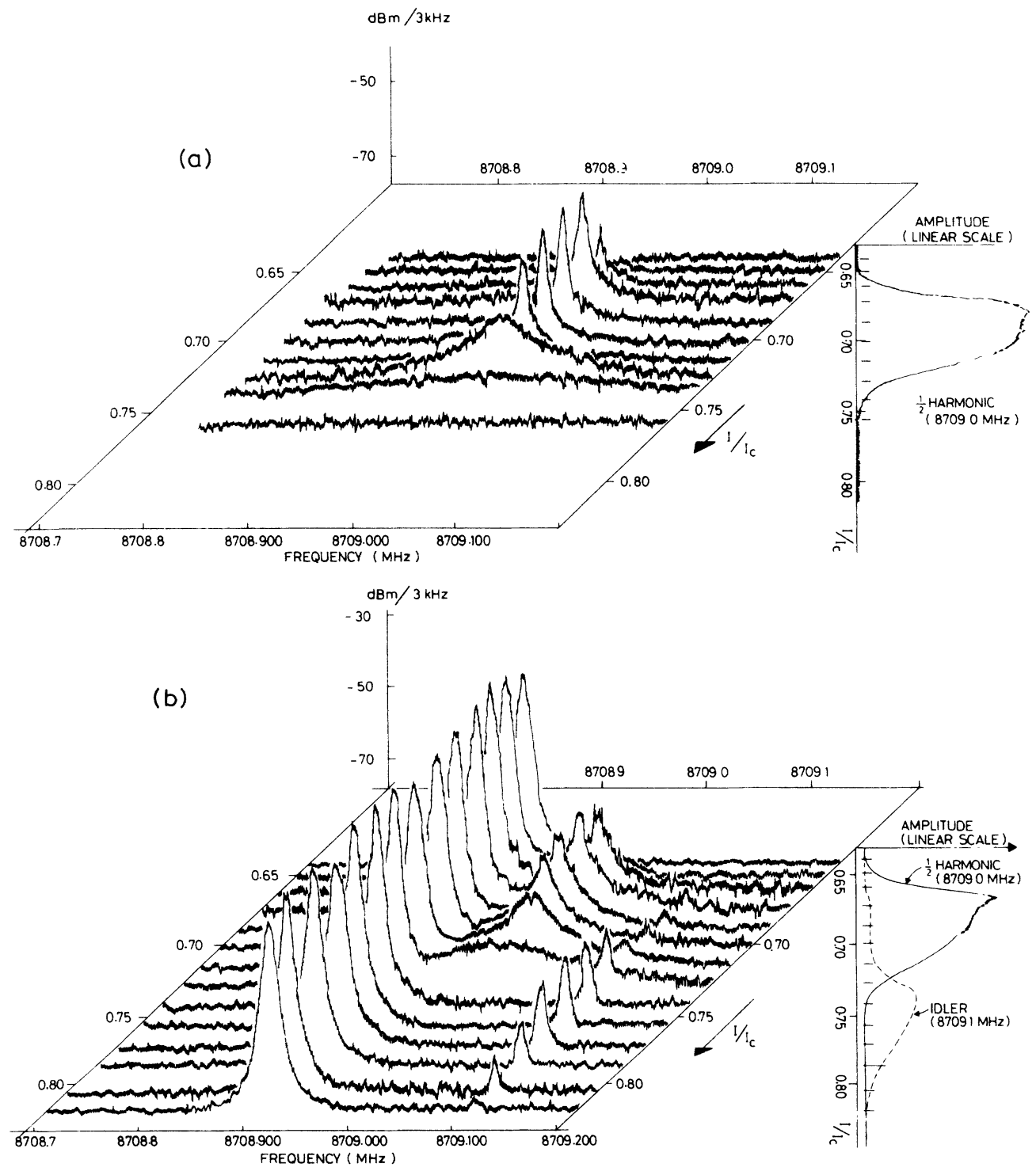

FIG. 3. Experimental data for a Nb-NbO $-\mathrm{Pb}$ tunnel junction showing the spectrum analyzer output vs the dc bias both (a) without a perturbation signal applied and (b) with an applied perturbation signal detuned by $-0.1 \mathrm{MHz}$ from $\omega_{D} / 2=2 \pi \times 8709.000$ MHz. The maximum amplitudes at $\omega_{D} / 2$ and at the idler (image) frequency at $2 \pi \times 8709.100 \mathrm{MHz}$ are also shown as functions of the dc bias. Both the suppression of the half-harmonic signal and the amplification are seen in this figure. 
Figure 4 shows how the onset of the period-doubled signal is shifted towards higher values of the drive amplitude when the amplitude of the perturbation signal is increased, i.e., the bifurcation point is moved towards larger $A_{D}$ values.

All the data reported here was taken for junctions biased in the zero-voltage state (on the supercurrent). With a large applied magnetic field of the order of $10 \mathrm{Oe}$ the same behavior was observed when junctions were biased on resonant cavity-mode current singularities in the $I-V$ curve ("Fiske" steps).

To find a possible characteristic scaling behavior of the shift of the bifurcation point we again use the amplitude of the drive signal as the nonlinearity parameter, $\mu$. From the experimental curves like the set reproduced in Fig. 4 we calculated the shift of the bifurcation point $\Delta \mu_{B}=\Delta A_{D}$ as a function of the perturbation amplitude $A_{B}$.

In Fig. 5 the bifurcation point shift found in this way is plotted versus the perturbation amplitude for three different values of the frequency detuning. From such double-logarithmic plots we have found a linear $\Delta \mu_{B}$ versus $A_{S}$ dependence for small detuning and a quadratic scaling behavior for large detuning: $\Delta \mu \sim A_{S}^{2}$.

Finally, for fixed $\Delta \mu_{B}$ we have investigated how the necessary perturbation amplitude depends on the detuning. It turns out that such measurements are very difficult to perform on a Josephson junction due to the strong frequency dependence of the microwave coupling to the junction. Technically it is not easy to vary the perturbation frequency over a wide band, say from 0.1 to 100 $\mathrm{MHz}$ (corresponding to a relative detuning from $10^{-5}$ to $10^{-3}$ ) without strongly varying the perturbation amplitude in the junction. At present we have therefore not been able conclusively to test the theoretical prediction for the scaling law for $A_{S}(\delta)$.

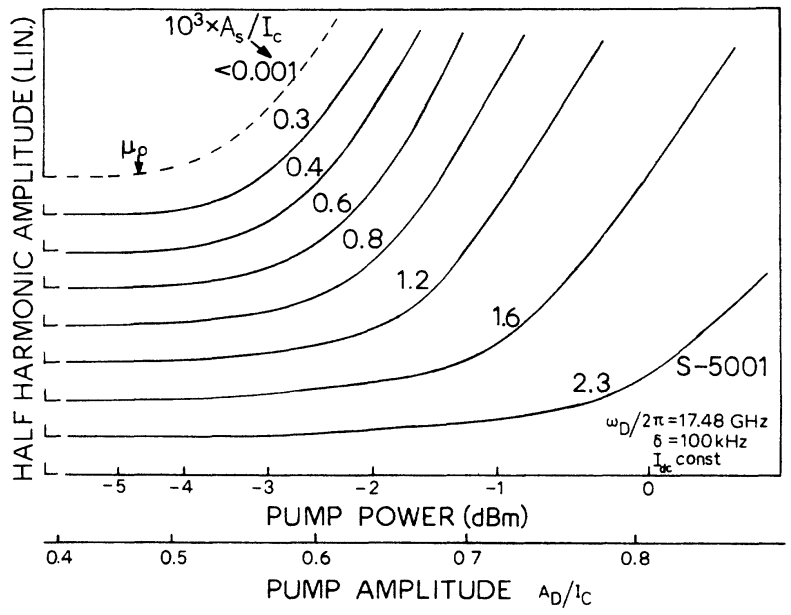

FIG. 4. Smoothed experimental recordings of the halfharmonic amplitude (linear scale) vs the normalized drive amplitude, $A_{D} / I_{c}$, for a number of different values of the normalized perturbation signal amplitude, $A_{S} / I_{c}$. The dc bias is constant, $\mu_{0}$ is the unperturbed bifurcation point, i.e., the threshold for the onset of the period-doubled signal (the half-harmonic) without perturbation.

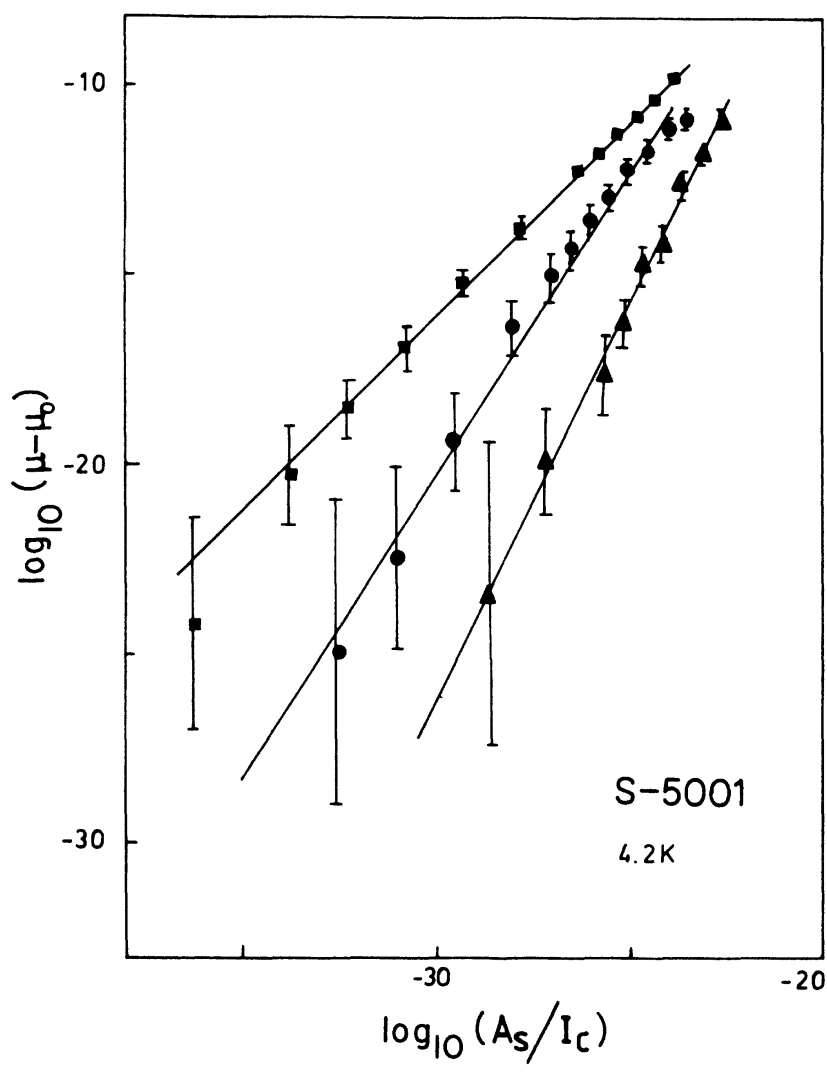

FIG. 5. Experimental data for a $\mathrm{Nb}-\mathrm{NbO}_{x}-\mathrm{Pb}$ tunnel junction at $4.2 \mathrm{~K}$. The log-log plot shows the measured scaling behavior of the shift of the bifurcation point (as defined by the minimum drive amplitude for which the period-doubled signal is observed; see Fig. 4) as a function of the normalized perturbation amplitude, $A_{S} / I_{c}$, for three different values of the detuning $\delta$ : squares $0.1 \mathrm{MHz}$, circles $10 \mathrm{MHz}$, and triangles $40 \mathrm{MHz}$. The value of $\omega_{D} / 2$ is $2 \pi \times 8709.000 \mathrm{MHz}$. The three straight lines are least-squares fits to the data points. The slopes give the following scaling exponents: squares 1.0, circles 1.6, and triangles 2.1.

\section{DISCUSSION AND CONCLUSION}

The stabilizing effect of a near-resonant perturbation signal against the onset of a period-doubling bifurcation that is predicted by the theory and confirmed through numerical calculations and analog simulations has been observed in small Josephson tunnel junctions biased on the supercurrent. Theoretically, the strength of this stabilization, which may be characterized by the shift of the bifurcation point, has been shown to depend on the perturbation amplitude according to a characteristic quadratic scaling law, i.e., $\Delta \mu_{B} \approx A_{S}^{2}$, where we have used the change in the drive amplitude, $\Delta A_{D}$, as a measure of the shift of the bifurcation point (i.e., we use $A_{D}$ as the nonlinearity parameter of the system).

In the experiments the shift in the bifurcation point was measured by the change of the minimum value of the drive amplitude for which a period-doubled signal could be observed above the background noise level in the sys- 
tem (Fig. 4). This shift was found to scale linearly with the perturbation amplitude for small-frequency detuning and quadratically for large detuning of the perturbation signal (Fig. 5). The same behavior was found in the numerical calculations (see Fig. 4 of Ref. 1). The junction is most sensitive to the perturbation signal for small detuning, and a large detuning gives a small value of the relative perturbation amplitude $A_{S} / \delta$. For small values of $A_{S} / \delta$ our experimental and numerical results both give a quadratic scaling law $\Delta \mu_{B} \approx A_{S}^{2}$.

\section{ACKNOWLEDGMENTS}

We thank I. Rasmussen, S. Hjorth, and J. L. Skov for the sample fabrication and T. Holst for the analysis of the experimental noise data. This work was partly supported by grants from the Danish Natural Science Research Council.
${ }^{1}$ Kurt Wiesenfeld and Bruce McNamara, Phys. Rev. Lett. 55, 10 (1985); Phys. Rev. A 33, 629 (1986); Paul Bryant and Kurt Wiesenfeld, ibid. 33, 2525 (1986); and Kurt Wiesenfeld, J. Stat. Phys. 28, 1071 (1985).

${ }^{2}$ Henrik Svensmark, J. Bindslev Hansen, and N. F. Pedersen, Phys. Rev. A 35, 1457 (1987).

${ }^{3}$ H. Svensmark and M. R. Samuelsen, Phys. Rev. A 36, 2413
(1987).

${ }^{4}$ H. Svensmark and M. R. Samuelsen, preceding paper, Phys. Rev. B 41, 4180 (1990).

${ }^{5}$ A. D. Smith, R. D. Sandell, J. R. Burch, and A. H. Silver, IEEE Trans. Magn. MAG-21, 1022 (1987).

${ }^{6}$ H. K. Olsson and T. Claeson, Jpn. J. Appl. Phys. 26, 1547 (1987). 